\title{
Atomic Motion Observed with the IBM Sub-Angstrom STEM
}

\author{
P.E. Batson
}

\section{IBM T.J. Watson Research Center, Yorktown Heights, New York 10598}

The IBM $120 \mathrm{kV}$ VG Microscopes HB501 STEM is currently able to produce a $0.8 \AA$ spot using the first Nion 2nd generation quadrupole-octupole aberration corrector. [1, 2] This performance is possible using several strategies to optimize the optics of the original configuration: 1) The field emission tip-extraction anode distance is controlled to an accuracy of $0.1 \mathrm{~mm}$, and the extraction voltage is kept within a range of $3.7-5 \mathrm{kV}$. As summarized in Figure 1, this minimizes the magnification of the source size within the gun, thereby indirectly increasing flexibility in operation of the corrector. This requirement is exacerbated by operation at $120 \mathrm{kV}$, where tele-focal conditions can be obtained for small extraction voltages. At $100 \mathrm{kV}$ with a $2 \AA$ probe size, the source size contribution was never the performance limiting factor, and so it has not been controlled before. 2) Adjustment of the condensor lenses can then match a fairly large $(40-50 \mu \mathrm{m})$ virtual objective aperture to a probe convergence of $45-48 \mathrm{mR}$. 3) The size of the beam within the corrector is adjusted for best aberration correction. This process can reduce the combination aberration $\mathrm{C}_{5}$ to about 3-5 cm by, in effect, introducing a weak transfer lens between the $\mathrm{C}_{3}$ correction planes and the back focal plane of the objective lens. The effectiveness of this appears to be limited by large angle distortions introduced as the beam becomes very large. Thus, this system has a theoretical limiting probe size of $0.31-0.43 C_{5}^{1 / 6} \lambda^{5 / 6}=0.5-0.7 \AA$. The measured probe size is $0.8-0.9 \AA$ with a probe current of $50-75 \mathrm{pA}$.

This large probe current allows STEM ADF images to be obtained at 5-10 fps using a National Instruments PC-1409 image acquisition board set up to acquire 1024x1024 pixel 10 bit images. Figure 2 shows an example of an $\mathrm{Si}_{-} \mathrm{SiO}_{2}$ interface in a $\mathrm{Hi}-\mathrm{K}$ dielectric gate structure. It is well known that an amorphous Hf silicate decomposes on heating into Hf-rich and Hf-poor regions. After decomposition, Hf atoms are also present near the oxide-Si interface and move very easily under the $120 \mathrm{kV}$ beam. Single atoms of Hf are easily followed for short distances. Often, movement consists of no motion for 2-3 frames at $5 \mathrm{fps}$, followed by a large displacement away from the field of view. Occasionally a single atom can be followed for several frames. In this case, Hf atoms appear to explore a neighborhood that is about 4-5 $\AA$ wide, running parallel to the [001] silicon plane. I speculate that this movement may be associated with a quasi-planar network of $\mathrm{Si}$ atoms within the amorphous $\mathrm{SiO}_{2}$. Movement is not apparent in the $\mathrm{Si}$ or $\mathrm{HfO}_{2}$ under this relatively low energy beam. Although movement under the beam would seem to be an undesirable indication of radiation damage, the relative movement of different kinds of atoms does help us to form predictions about stability of the final structure.

[1] N. Dellby, O.L. Krivanek, P.D. Nellist, P.E. Batson, and A.R. Lupini, J. Electron Microscopy, 50 (2001)177 $-185$.

[2] P.E. Batson, Niklas Dellby, and O.L. Krivanek, Nature, 418 (2002)617 - 620. 


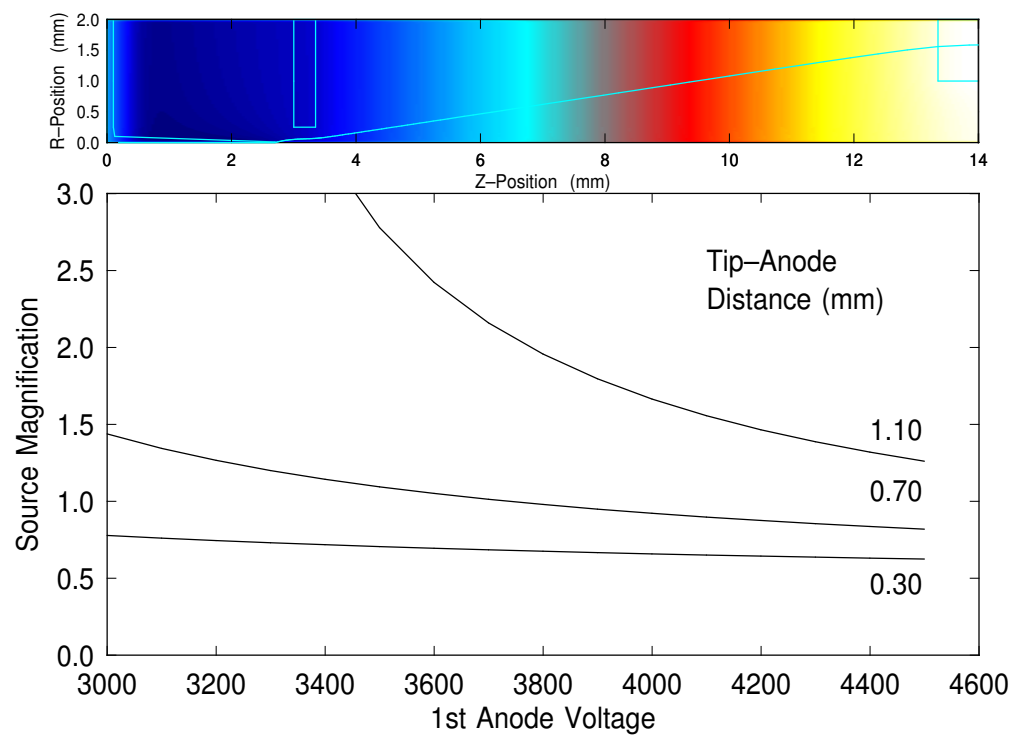

Fig. 1. Summary of optical calculation for the VG gun operating at $120 \mathrm{kV}$. For a tip-anode distance of $0.5-0.6 \mathrm{~mm}$, the source magnification is always less than 1.6x. Cs and Cc remain less than $0.05 \mathrm{~mm}$.

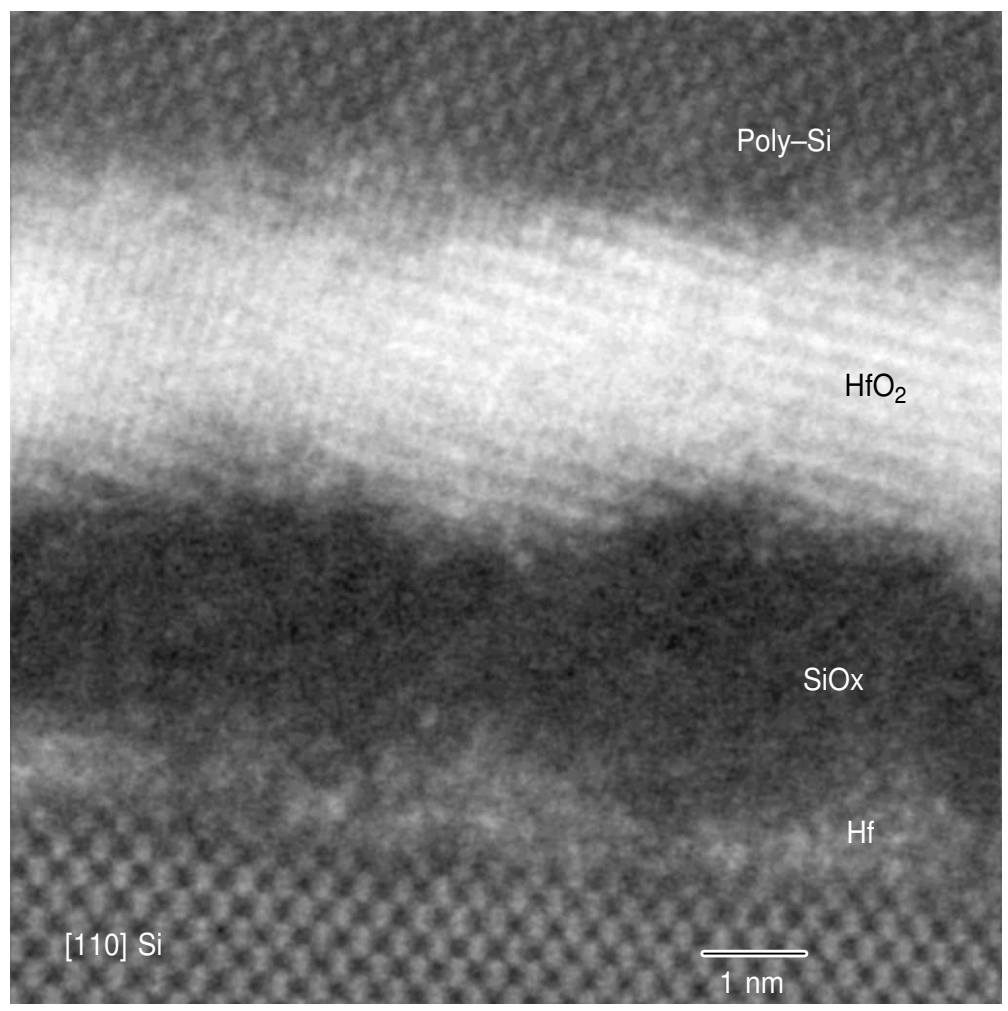

Fig. 2. Oxide dielectric structure, formed by annealing amorphous hafnium silicate on $\mathrm{SiO}_{2}$ and silicon. Single Hf atoms can be followed. 